\title{
Factitious Cushing's syndrome, hypopituitarism, and self-provoked skin lesions: when the skin mirrors the soul
}

\author{
Salvatore Cannavò ${ }^{1}$ and Serafinella Patrizia Cannavò ${ }^{2}$ \\ 1 Unit of Endocrinology of Department of Human Pathology and 2Unit of Dermatology of Department of Clinical and \\ Experimental Medicine, University of Messina, Messina, Italy
}

Correspondence should be addressed to S Cannavò Email

cannavos@unime.it

\section{Summary}

Factitious Cushing's syndrome (CS) is a very rare form of Münchausen syndrome. Its presentation and course are extremely heterogeneous, and diagnosis is generally challenging. We report the case of a 52-year-old woman who was initially investigated because of the occurrence of cushingoid features. Nevertheless, endocrine work-up showed very low morning plasma ACTH and serum cortisol levels. In addition, it also demonstrated central hypopituitarism and hypogonadotropic hypogonadism. Head MRI showed a small pituitary mass. Based on these results, and probably overlooking the initial clinical suspicion, general practitioner (GP) referred the patient to our Endocrine Unit for hypopituitarism. At inspection, moon face, central obesity, and bruising were evident. Multiple ulcerative skin lesions were also concentrated in the right arm and leg. Dermatology evaluation suggested that the lesions were self-provoked. For several days, the patient denied the assumption of corticosteroids, but we finally discovered that the GP' nurse had prescribed betamethasone without the GP's knowledge for about 2 years. In conclusion, the surreptitious assumption of corticosteroids is very rare, but the physicians should be aware that pituitary function could be impaired by high doses of corticosteroids, mimicking hypopituitarism. In these patients, a multidisciplinary approach and environmental investigation can be useful to diagnose factitious CS.

\section{Learning points:}

- Surreptitious assumption of corticosteroids can cause heterogeneous presentation, ranging from Cushing's syndrome to multiple hypopituitarism.

- Suppression of ACTH and cortisol levels in a patient with cushingoid features firstly suggests surreptitious assumption of corticosteroids.

- A multidisciplinary approach can be extremely useful in patients with suspected factitious Cushing's syndrome.

- Sometimes, to prove surreptitious assumption of corticosteroids needs environmental investigation.

\section{Background}

Surreptitious use of drugs is a relatively frequent psychiatric disturbance, known as Münchausen syndrome (1). It causes factitious physical and psychological alterations that are, at the same time, the goal and the gain for which the patient takes the drug. In most cases, patients are young women, with a history of depression or anxiety, sometimes previous surgery, and a medical-related occupation or close contact with a medical worker (2). For instance, surreptitious assumption of antidiabetic drugs or self-administration of insulin is well-known causes of hypoglycemia in nondiabetic subjects (3). 
Corticosteroids are among the most prescribed drugs worldwide, and their chronic use at high doses for the treatment of non-endocrine diseases is the most frequent cause of Cushing's syndrome (CS). On the contrary, endogenous hypercortisolism is a rare condition that can be due to ACTH-stimulated or primary adrenal hypersecretion (4). Despite steroids abuse is extremely frequent, only a few cases of factitious CS were reported until now (5). Presentation and course of factitious CS are extremely heterogeneous. Indeed, some patients show typical signs and symptoms of hypercortisolism, while other ones are asymptomatic or complain of symptoms due to adrenal insufficiency (AI), caused by long-term inhibition of hypothalamic-pituitary-adrenal axis. Diagnosis is generally challenging, and several expensive or invasive approaches are frequently performed to prove the surreptitious assumption of corticosteroids (6).

Here, we present the intriguing case of a woman with cushingoid features, a small pituitary mass detected by MRI, and biochemical profile indicating multiple hypopituitarism, in whom the diagnosis of factitious CS was suspected because of the occurrence of self-induced skin lesions, and finally confirmed serendipity.

\section{Case presentation}

A 52-year-old woman with cushingoid features was referred to our Endocrine Unit from her general practitioner (GP). Endocrine investigation had paradoxically shown undetectable morning levels of serum cortisol and plasma ACTH. Central hypothyroidism and hypogonadotropic hypogonadism were also evident (Table 1 ). In addition, a 4-mm hypointense lesion in the anterior pituitary gland had been detected by MRI. Hypertension and dyslipidemia were treated for 13 years, and she had complained of myalgia, headache resistant to analgesic drugs, asthenia, and fatigue for the last 3 years. One year before, she had been admitted to the Endocrine Unit of another university hospital for some weeks. During this period, the initially very low ACTH and cortisol levels spontaneously and progressively increased, until complete normalization. Unfortunately, the patient did not show documents of this hospitalization reporting on conclusions or diagnosis.

\section{Investigation}

At entry, blood pressure was 90/60 $\mathrm{mmHg}$, and heart rate was 96 b.p.m, but she showed evident cushingoid features, including central obesity (BMI was $29 \mathrm{~kg} / \mathrm{m}^{2}$, waist/hip ratio 1.06), moon face, sarcopenia, and bruising.
Table 1 Biochemical profile of patient, at entry and at last evaluation, after appropriate corticosteroids titration.

\begin{tabular}{|c|c|c|c|}
\hline Laboratory report & Result at entry & Last result & Normal value \\
\hline $\begin{array}{l}\text { Serum morning } \\
\text { cortisol }(\mu \mathrm{g} / \mathrm{dL})\end{array}$ & 0.7 & 17.8 & $5-25$ \\
\hline $\begin{array}{l}\text { Plasma morning } \\
\text { ACTH }(\mathrm{pg} / \mathrm{mL})\end{array}$ & $<5$ & 23.2 & $10-50$ \\
\hline $\begin{array}{l}\text { Urinary free cortisol } \\
(\mu \mathrm{g} / 24 \mathrm{~h})\end{array}$ & 35.2 & 303 & $30-350$ \\
\hline DHEAS $(\mu \mathrm{g} / \mathrm{dL})$ & $<2$ & 12.5 & $35-430$ \\
\hline $\mathrm{FSH}(\mathrm{IU} / \mathrm{L})$ & 2.4 & 41 & $17-114$ \\
\hline LH (IU/L) & 0.2 & 23 & $11-59$ \\
\hline TSH (mU/L) & 1.2 & 1.3 & $0.3-4.2$ \\
\hline $\mathrm{E} 2(\mathrm{pg} / \mathrm{mL})$ & 11.6 & $<5$ & $<47$ \\
\hline FT4 (pM/L) & 11.1 & 13.4 & $12-22$ \\
\hline PRL (IU/L) & 206 & 274 & $58-416$ \\
\hline Lymphocytes (\%) & 28 & 35 & - \\
\hline Sodium (mMol/L) & 139 & 145 & $135-148$ \\
\hline Potassium (mMol/L) & 4.1 & 4.6 & $3.5-5.2$ \\
\hline Glycemia (mg/dL) & 83 & 69 & $65-110$ \\
\hline
\end{tabular}

Nevertheless, the patient resolutely denied the assumption of corticosteroids or herbal preparations, and steroids administration through food or other drugs - even without the patient's knowledge - was absolutely ruled out. Her husband confirmed that the patient did not take corticosteroids, despite our insistent requests. In addition, the inspection of skin revealed multiple ulcerative lesions, with a clear base and well-defined, indurated margins, prevalent in the right side of the body (Fig. 1). The ulcers were surrounded by clear skin, even though it appeared diffusely thinner and anelastic. Interestingly, these lesions were concentrated on the right side of the body, especially on the right leg. We suspected that skin lesions were selfprovoked also because the patient was left-handed and could not exactly pinpoint when they first appeared, but psychiatric counseling excluded mental disorders, demonstrating only excessive hostility against physicians who had previously managed her.

\section{Treatment}

At this point, we consulted her GP by phone, to verify the clinical history of the patient and to check the therapies recently performed. He denied having frequently prescribed steroids but his nurse, present during the call, acknowledged that she had prescribed to the patient about 20 pills of betamethasone $1 \mathrm{mg}$, every week for about 2 years. At this point, we recommended to progressively decrease and finally withdraw betamethasone, to replace it with hydrocortisone $20 \mathrm{mg}$ orally every day, because of the persistent suppression of hypothalamic-pituitary- 

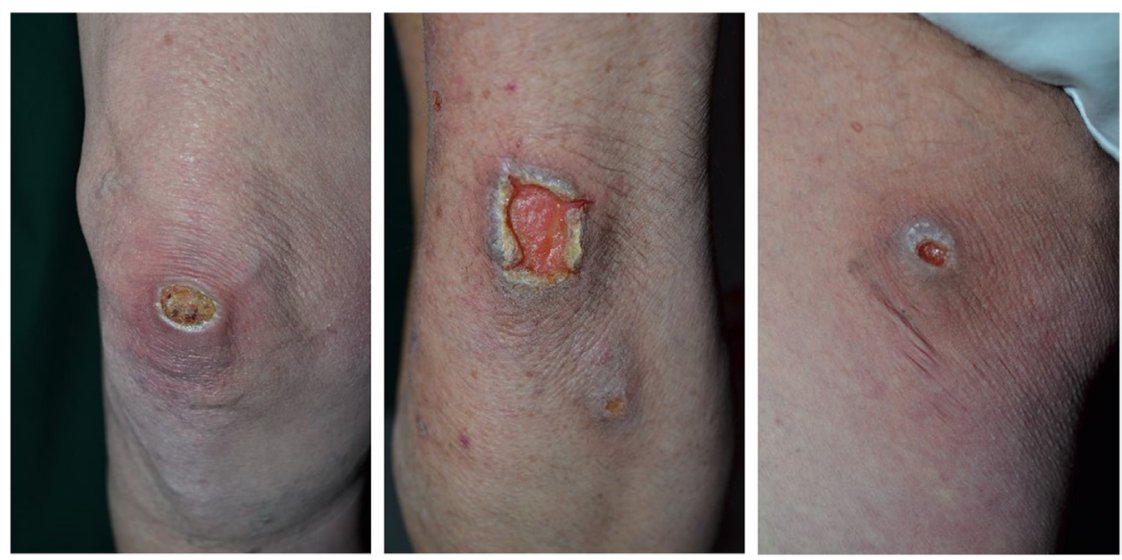

\section{Figure 1}

Self-provoked ulcerative lesions, localized on the right limbs (from left to right: knee, forearm, thigh). They had a clear base and well-defined, indurated margins and were surrounded by clear skin.

adrenal (HPA) axis, and to start levothyroxine substitution. In addition, psychiatric re-evaluation was performed, and treatment with serotonin reuptake inhibitor antidepressant (sertaline $50 \mathrm{mg}$ daily) was prescribed for 6 months. Skin lesions were treated by occlusive bandages and collagenase ointments.

\section{Outcome and follow-up}

The patient was re-evaluated after 8 months. Treatment with low-dose hydrocortisone was still maintained but, when we evaluated the HPA axis during a short wash-out of hydrocortisone, morning ACTH and cortisol levels were in the normal range. Levothyroxine administration was withdrawn because thyroid function was normalized. In addition, serum gonadotropins levels increased. Biochemical parameters measured at the last evaluation are shown in Table 1.

\section{Discussion}

Clinical consequences of surreptitious use of corticosteroids are extremely heterogeneous, ranging from typical CS to AI. Indeed, they are related to individual sensitivity to glucocorticoids and to length, doses, and route of drug administration (4). In a systematic review of 455 cases of factitious disorders, Yates and Feldman reported corticosteroids abuse in only 9 out of 59 patients with endocrine manifestations (2). In the other cases, hypoglycemia was the most frequent manifestation. Overall, a presentation unsubstantiated by objective clinical evidence was found to have facilitated the discovery of factitious disorder (2). Clinical history of our patient was in accordance with this suggestion. Indeed, exogenous CS was initially suspected not only because cushingoid features were in contrast with suppressed HPA axis and adrenal hypotrophy but also because of central hypothyroidism and inappropriately low gonadotropins levels cannot be justified by the occurrence of a very small pituitary lesion, generally unable to cause pituitary deficiency, while chronic glucocorticoid excess can suppress TSH and gonadotropins secretion. In addition, the occurrence of self-inflicted skin lesions suggested the diagnosis of Münchausen syndrome. Several years ago, Kansagara et al. reported on the case of a woman affected by factitious Cushing's syndrome and died for invasive aspergillosis, who presented numerous ulcerated lesions, ranging up to $3 \mathrm{~cm}$ in diameter, on the anterior chest and arms (7). In the aforementioned paper of Yates and Feldman, 43 patients showed self-induced dermatological lesions (2). They were generalized or localized especially into the breast, face, legs, or genitalia. These pathomimic lesions are generally characterized by constant picking of the skin, leading to bizarre-looking aspects, usually prevalent on easily accessible areas of the body, surrounded by normal skin (8). The patients, usually females on the onset of puberty or in the later ages, find a way to relieve stress and anxiety through the repeated trauma of the skin, leading to the worsening of the mental condition triggering this phenomenon. Often, they seem to be clueless on how and when these lesions appear. Skin biopsy is generally unnecessary, because the diagnosis is easy, especially for an expert eye. A history of depression is reported in many cases of factitious CS, but psychiatric evaluation can be negative or demonstrate only mild personality disorders as in our patient (9). On the other hand, anxiety, major depression, and bipolar disorders are frequently observed in patients with endogenous CS (4). Chronic brain exposure to cortisol excess causes deep structural and functional changes in various cerebral areas that are rich in glucocorticoid receptors, particularly the hippocampus, amygdala, and prefrontal cortex, including the limbic system, all of which are fundamental regions 
for emotional and cognitive functions. For this reason, a differential diagnosis between primary or secondary psychiatric disturbances can be challenging in these patients. The case reported by Kansagara et al. also underlines that patients exposed to chronic glucocorticoids excess undergo increased morbidity and mortality risk due to infectious disease (7). It is largely demonstrated by studies on endogenous CS, and it should be remembered also in the management of patients with factitious CS (4). Indeed, purulent lesions, lichenification, s.c. abscesses or pyoderma gangrenosum can occur (2).

Sometimes, to demonstrate surreptitious corticosteroids assumption can be difficult and frustrating. Azizi et al. reported on the occurrence of factitious CS in 19 narcotic addicts using a black-market drug for narcotic withdrawal, in which buprenorfin had been replaced by dexamethasone and heroin (5). In other cases, relatives do not collaborate with physicians to discover the truth because they are accomplices of the patient. This was the case reported by Kansagara et al., in which a criminal investigation demonstrated that the husband was guilty of aiding the patient in illegally securing corticosteroids (7). Also in our case, it was inconceivable that the husband of the patient was unaware of her corticosteroids abuse. Nevertheless, we would not demonstrate the surreptitious assumption of bethamethasone if the patient's GP had not been involved in our investigation and if its nurse had not cooperated.

As well as clinical features, also the biochemical profile of the HPA axis can be extremely heterogeneous in patients with factitious CS. Indeed, plasma ACTH and serum or urinary cortisolvalues are very low in somecases, but normal or increased in other ones $(6,10,11)$. In some patients, the biochemical profile mimics Cushing's disease, and MRI can incidentally detect small pituitary lesions (12). Several explanations were proposed for this variability: regimen and doses of surreptitious corticosteroids assumption, type of steroids (e.g. prednisolone or betamethasone), sensitivity of immunoassays used for ACTH, and cortisol measurement and cross-reactivity with other steroids. In many cases, steroids measurement by LC-MS/MS allowed a correct diagnosis, but it followed invasive, expensive, and sometimes unnecessary procedures. For instance, the investigation included adrenal scintigraphy, inferior petrosal sinus sampling, PET, or finally NR3C1 gene sequencing, in some patients $(6,9,10,13)$. The physicians should be aware that the risk of misdiagnosis in presence of incidentally discovered pituitary or adrenal masses could be very high, especially if the HPA axis is not suppressed, but also that pituitary function can be impaired by high doses of corticosteroids.

Small pituitary masses are incidentally diagnosed by MRI, or at autopsy, in about $10 \%$ of the general population. When it occurs in patients with cushingoid features, but without overt biochemical hypercortisolism, to rule out cyclic Cushing's disease, or at least partial apoplexy of a corticotroph tumor, could be difficult and time-consuming. Frequently, the endocrine workup needs to be repeated several times before to demonstrate hypercortisolism. Nevertheless, it is extremely rare to find suppressed HPA axis in these cases. In addition, exacerbation of signs and symptoms and increase of ACTH and cortisol levels generally occur simultaneously. Inferior petrosal sinus sampling, with bilateral basal and CRH-stimulated ACTH simultaneous measurement, can help to perform the correct diagnosis in challenging cases (14).

In conclusion, factitious CS occurs rarely, but its diagnosis is frequently challenging. Surreptitious assumption of corticosteroids can impair pituitary function, also mimicking hypopituitarism. A multidisciplinary approach and environmental investigation can be useful especially in these patients for the appropriate interpretation of their clinical features.

\section{Declaration of interest}

The authors declare that there is no conflict of interest that could be perceived as prejudicing the impartiality of the research reported.

\section{Funding}

This work did not receive any specific grant from any funding agency in the public, commercial or not-for-profit sector.

\section{Patient consent}

Written informed consent has been obtained from the patient for publication of the submitted article and accompanying images.

\section{Author contribution statement}

Cannavò S has written the paper. Cannavò S P has reviewed the manuscript. Cannavò S and Cannavò S P are the endocrinologist and the dermatologist, respectively, who managed the patient and performed the diagnosis.

\section{References}

1 Schrader H, Bohmer T \& Aasly J. The incidence of diagnosis of Munchausen syndrome, other factitious disorders, and malingering. Behavioural Neurology 20192019 3891809. (https://doi. org/10.1155/2019/3891809) 
2 Yates GP \& Feldman MD. Factitious disorder: a systematic review of 455 cases in the professional literature. General Hospital Psychiatry 2016 41 20-28. (https://doi.org/10.1016/j.genhosppsych.2016.05.002)

3 Kittah NE \& Vella A. MANAGEMENT OF ENDOCRINE DISEASE: Pathogenesis and management of hypoglycemia. European Journal of Endocrinology 2017177 R37-R47. (https://doi.org/10.1530/EJE-16-1062)

4 Pivonello R, Isidori AM, De Martino MC, Newell-Price J, Biller BMK \& Colao A. Complications of Cushing's syndrome: state of the art. Lancet: Diabetes and Endocrinology 20164 611-629. (https://doi. org/10.1016/S2213-8587(16)00086-3)

5 Azizi F, Jahed A, Hedayati M, Lankarani M, Bejestani HS, Esfahanian F, Beyraghi N, Noroozi A \& Kobarfard F. Outbreak of exogenous Cushing's syndrome due to unlicensed medications. Clinical Endocrinology 200869 921-925. (https://doi.org/10.1111/j.13652265.2008.03290.x)

6 Thynne T, White GH \& Burt MG. Factitious Cushing's syndrome masquerading as Cushing's disease. Clinical Endocrinology 201480 328-332. (https://doi.org/10.1111/cen.12343)

7 Kansagara DL, Tetrault J, Hamill C, Moore C \& Olson B. Fatal factitious Cushing's syndrome and invasive aspergillosis: case report and review of literature. Endocrine Practice 200612 651-655. (https://doi. org/10.4158/EP.12.6.651)

8 Chiriac A, Foia L, Birsan C, Goriuc A \& Solovan C. Cutaneous factitia in elderly patients: alarm signal for psychiatric disorders. Clinical Interventions in Aging 20149 421-424. (https://doi.org/10.2147/CIA. S56185)

9 Pineyro MM, Redes L, De Mattos S, Sanchez L, Brignardello E, Bianchi V, Ems V, Centurión D \& Viola M. Factitious Cushing's syndrome: a diagnosis to consider when evaluating hypercortisolism. Frontiers in Endocrinology 201910 129. (https://doi.org/10.3389/ fendo.2019.00129)

10 Kline GA, Buse JD, Van Der Gugten JG, Holmes DT, Chin AC \& Sadrzadeh SMH. Factitious ACTH-dependent, apparent hypercortisolism: the problem with late-night salivary cortisol measurements collected at home. Clinical Endocrinology 201787 882-885. (https://doi.org/10.1111/cen.13478)

11 Ach K, Khochtali I, Ajmi ST, Beizig AM, Chadli MC, Ajina MZ \& Chaieb L. Factitious Cushing syndrome: two case reports. La Revue de Médecine Interne 200526 973-976. (https://doi.org/10.1016/j. revmed.2005.07.011)

12 Sakai H, Matsumoto K, Sugiyama M, Yoshimoto T, Doi M, Izumiyama H \& Hirata Y. A case of factitious adrenal insufficiency after vascular graft surgery caused by spurious immunometric assays. Endocrine Journal 200653 415-419. (https://doi.org/10.1507/endocrj. k06-006)

13 Villanueva RB, Brett E \& Gabrilove JL. A cluster of cases of factitious Cushing's syndrome. Endocrine Practice 20006 143-147. (https://doi. org/10.4158/EP.6.2.143)

14 Pecori Giraldi F, Cavallo LM, Tortora F, Pivonello R, Colao A, Cappabianca P, Mantero F \& Altogether to Beat Cushing's Syndrome Group. The role of inferior petrosal sinus sampling in ACTH-dependent Cushing's syndrome: review and joint opinion statement by members of the Italian Society for Endocrinology, Italian Society for Neurosurgery, and Italian Society for Neuroradiology. Neurosurgical Focus 201538 E5. (https://doi. org/10.3171/2014.11.FOCUS14766)

Received in final form 16 July 2021 Accepted 26 July 2021 